\title{
BeiDou 3 Signal Quality Analysis and its Impact on Users
}

\author{
Steffen Thoelert ${ }^{1,2}$, Felix Antreich ${ }^{3}$, Christoph Enneking ${ }^{1}$, Michael Meurer ${ }^{1,2}$ \\ ${ }^{1}$ German Aerospace Center (DLR), Oberpfaffenhofen, 82234 Wessling, Germany \\ ${ }^{2}$ Chair of Navigation, RWTH Aachen University, 52074 Aachen, Germany \\ ${ }^{3}$ Instituto Tecnológico de Aeronáutica (ITA), São José dos Campos, SP 12228-900, Brazil
}

\section{BIOGRAPHIES}

Steffen Thoelert received his diploma degree in electrical engineering from the University of Magdeburg in 2002. In May 2006 he joined the Institute of Communications and Navigation at the German Aerospace Centre (DLR), Oberpfaffenhofen. His current research activities include signal quality monitoring, satellite payload characterization, system calibration, GNSS compatibility and interference aspects and remote sensing.

Felix Antreich received the Diploma degree in electrical engineering from the Munich University of Technology (TUM), Munich, Germany, in 2003. In 2011 he also received the Doktor-Ingenieur (Ph.D.) degree from the TUM. Since September 2018 he is a professor at the Instituto Tecnológico de Aeronáutica (ITA), São José dos Campos, Brazil

Christoph Enneking received the BSc. and the MSc. degrees in electrical engineering from the Munich University of Technology (TUM), Germany, in 2012 and 2014, respectively. In September 2014, he joined the Institute of Communications and Navigation of the German Aerospace Center (DLR), Wessling-Oberpfaffenhofen. His research interests include GNSS signal design, estimation theory, and GNSS intra- and intersystem interference.

Michael Meurer received the diploma in Electrical Engineering and the Ph.D. degree from the University of Kaiserslautern, Germany. Since 2006 he is the director of the Department of Navigation of the German Aerospace Center (DLR). In addition, he is a professor of electrical engineering and director of the Chair of Navigation at the RWTH Aachen University. His current research interests include GNSS signals, GNSS receivers, and navigation for safety-critical applications.

\begin{abstract}
Nowadays, one can use four global navigation satellite systems (GNSS). Two of them are complete constellations (GPS, Glonass) and two (BeiDou, Galileo) are already providing initial services and will be finished in the near future. Additionally, satellite-based augmentation systems (SBAS) and regional systems like WAAS, EGNOS, GAGAN or QZSS complement the GNSS service. However, within all systems one can observe changes, modifications, and updates every year. This can be related to signal property changes, renewing satellites up to the implementation of completely new GNSS platforms in space. Especially, for safety critical applications using GNSS, like advanced receiver autonomous integrity monitoring (ARAIM) or ground-based augmentation systems (GBAS) the new or changed signal properties are of high interest and are crucial for integrity analysis. With the help of detailed information about the signal deformation and the received signal power it is possible to calculate realistic error bounds and consequently realistic protection level for these kinds of safety-critical applications.
\end{abstract}

This paper will present signal analysis results based on observations of the new BeiDou 3 signals appeared in the last two years. After a brief introduction of the measurement facility the basic analysis of the quality of the signals in spectral and modulation domain is introduced. This covers the transition from BeiDou 2 up to BeiDou 3 including the test and validation phase in the beginning of BeiDou 3. Based on captured in-phase and quadrature (I/Q) samples 
we will give a detailed overview on the BeiDou 3 satellite payload characteristics including signal deformation analysis. The paper will assess how such imperfections will influence the pseudo-range measurements and consequently provide the capability for error analysis with respect to safety-critical applications. We will show the dependency of tracking biases depending on different receiver configuration.

Using the German Aerospace Center 's (DLR) precise calibrated measurement facility, we will also present an analysis of the transmitted satellite signal. Considering the measured power in relation to the boresight angle of the satellite one get a cut through the antenna pattern of the satellite and can assess the antenna symmetry properties. Examples for different satellites will be presented.

\section{INTRODUCTION}

The Chinese BeiDou navigation satellite system (BDS) is one of the most rapidly growing satellite navigation systems of the last years. It started 2000 with a demonstration phase with 3 geostationary test platforms. In a second step it has been expanded to a regional navigation system with 16 space crafts in total [Yang 2017]. In 2015 the Chinese have started updating their system from a regional to a global one with the launch of their first BeiDou 3 satellite. This development to a global system implies not only the expansion of the coverage but also a significant change of the provided signals and services to achieve better compatibility to existing GNSS and consequently less implementation effort at manufacturer side and therefore better competitiveness.

Until mid of 2017 the information about the BeiDou 3 signals and services were very fragmentary. From official statements and supplementary information which circulated in the navigation community, draft overviews with respect to service type, frequency and spreading modulations were collected and presented in [Betz 2015, Teunissen and Montenbruck 2017].

Figure 1 shows an extract of the GNSS signal overview of [Teunissen and Montenbruck 2017] regarding BeiDou, GPS, and Galileo. Clearly visible is the change in signal provision from a regional BeiDou system (BDS-2) to a global one (BDS-3). One can observe, that a certain similarity of the new BDS-3 signal frequencies and modulation types with GPS and Galileo is targeted which emphasize the ambitions for compatibility with other GNSS.

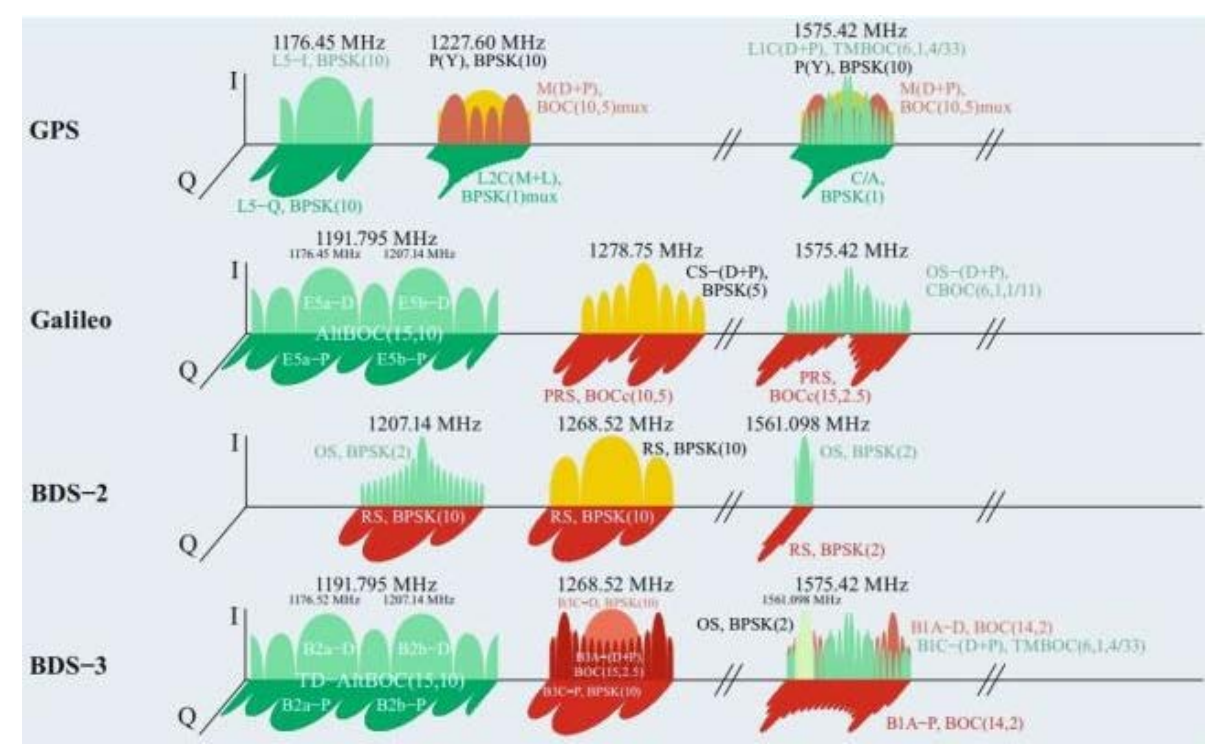

Figure 1 Signal overview regarding BeiDou 2 (regional navigation system) and BeiDou 3(global navigation system) and GPS and Galileo [Teunissen and Montenbruck 2017, page 1235]

Within the last 13 months interface control documents (ICDs) regarding the B1C, B3I and B2a have been released, which provide detailed information on signal structure, modulation, coding, message types, and structure. However, 
still a couple of signals without detailed official information exist, which motivates the authors at least to analyze the presence and modulation type of all L-band transmissions of BDS-3.

In March 2015 the first satellite BDS I1-S of BeiDou 3 was launched, followed by further four satellites, i.e. BDS M1-S, BDS M2-S, BDS I2-S and BDS M3-S. These first 5 initial BeiDou 3 satellites were some kind of test satellites for validation purposes [Betz 2015, page 262] but with operational capability. The satellites are indicated by an "S" in the end of each satellite name like BDS M2-S. The concept is similar to European Galileo implementation plan in the past - starting with the installation of the so-called Galileo In-Orbit Validation Elements (GIOVE A/B) followed by In-Orbit Validation (IOV) satellites with full operational capability (first four Galileo satellites). However, the Chinese have combined the former two steps into one. The analysis that is presented in the following will show that substantial tests have been performed and obviously the analysis resulted in certain changes of the final payload design.

Since 2017 China has already launched further 16 fully operational BeiDou 3 satellites. Therefore, now it seems to be the right time to analyze the present nominal state of the signal in space (SIS) behavior and to assess its usability for challenging GNSS applications, e.g. in the field of safety-critical applications.

The first signals of the new BeiDou 3 system were captured on August 10, 2015. Researchers from the Joint Research Center of the European Commission (JRC) at Ispra, Italy, captured the signal at B1 frequency (which corresponds to GPS L1 and Galileo E1 band) and conducted a first signal analysis in time, spectral, and correlation domain [Bravaro 2015]. Based on their analysis they confirmed the presence of a time-multiplexed binary offset carrier (TMBOC) signal and its power sharing among its individual components as well as -an additional BOC $(14,2)$ and a legacy B1I signal (BeiDou 2) at L1-14 MHz. Researchers from JAVAD GNSS and DLR also have been busy tracking the newest BeiDou satellites at that time, [Camaron 2015] summarized their findings. Researchers at JAVAD GNSS validated not only signals on L1 but also at E5a and E5b frequencies and provided a first impression on the signal strength which users can expect on ground. The researchers of DLR focused their analysis on spectral measurements to identify signals and their modulation type on all frequency bands in the overall L-band. Figure 1 provides an overall spectral overview of the transmitted signal on $24^{\text {th }}$ of October in 2015. The measured spectrum fits exactly with the expectation presented in Figure 1.

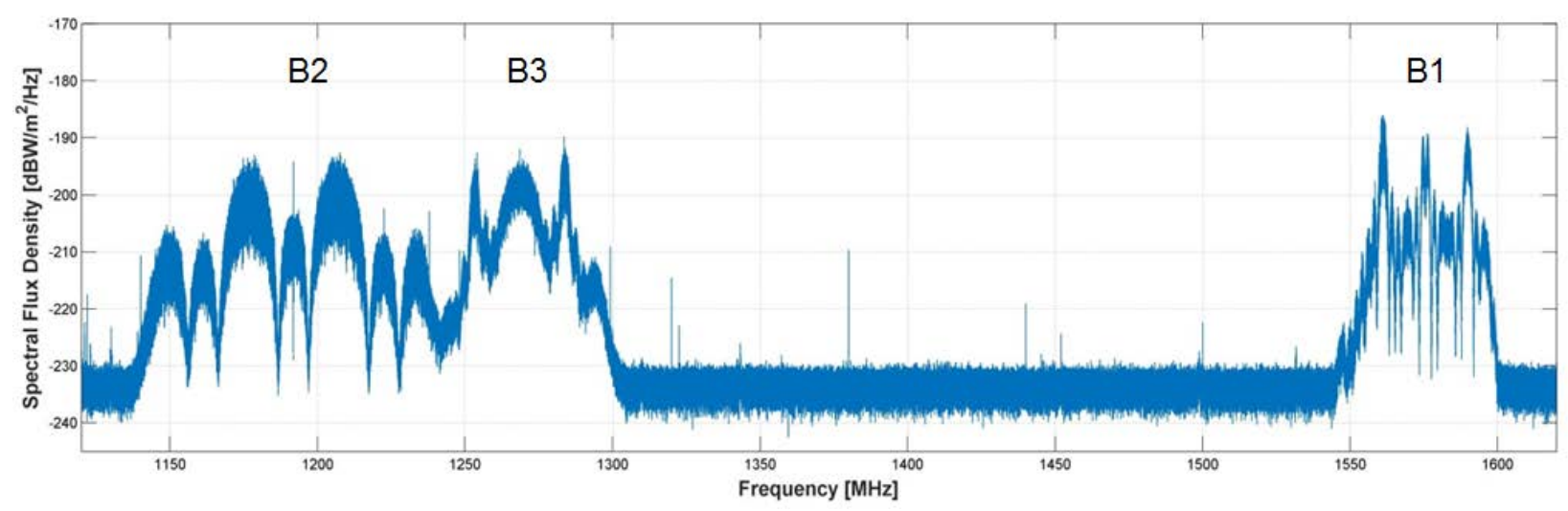

Figure 2 Spectral overview of the transmitted BeiDou L-band signals of BDS I2-S on 24.10.2015

In [Xiao 2016] receiver outputs and spectra are presented for all BeiDou 3 signals in the L-band. Additionally, inphase and quadrature data have been recorded and I/Q constellation diagrams are presented. However, with the included noise no statements about signal and modulation purity as well as quality could be retrieved.

Beside from research on the signal characteristics, scientists have been observed variations in the BDS resulting pseudo-ranges at user receiver level [Wu 2017, Zhou 2018]. Signal distortions are an important contribution to the 
signal in space range error (SISRE), hence motivating to perform very precise signal analysis to characterize qualitatively and quantitatively the amount and influence of the BeiDou 3 signal behavior.

Based on the above mentioned previous publications it can be concluded that they primarily addressed the investigation of basic modulation parameters of the signals and provided an initial analysis of the transmitted modulation type, chip rate, and primary as well as secondary codes. Detailed information about nominal signal distortions or information about signal power received on ground or antenna pattern characteristics are missing. However, such information is essential for the assessment of BeiDou 3 with respect to its usability for safety-critical applications.

This paper tries to fill the gaps of information on detailed and precise signal quality assessment to provide the basis for a usability analysis of BeiDou 3 regarding safety-critical applications. The analysis will be based on measurements with DLR's high gain antenna located in Weilheim, Germany. The SIS analysis part will contain significant more findings than in previous publications and will provide in-depth analysis of signal quality and signal distortions.

First, the DLR measurement facility and the necessary steps of data calibration will be briefly described. This accurate calibration is the key enabler for performing detailed measurements and allows for the compensation of the impact of the receiving system imperfections and propagation influences (e.g. ionospheric refraction).

After that, we will start with basic analysis of the quality of the signal shape in spectral and modulation domain. Based on the captured I/Q samples the authors will give a detailed overview on the BeiDou 3 satellite payload characteristics including analysis of signal distortions.

In order to assess the effect of such signal imperfections on safety-critical applications, the paper will assess how such errors propagate to the receiver observables. Setting out from the requirements and needs of these critical applications as well as current Minimum Operational Performance Standards (MOPS) the estimated signal characteristics and payload imperfections will be used to assess their impact on pseudo-range measurements. We will show the first time for the BeiDou 3 system the dependency of tracking biases on the receiver configurations. Much to a surprise is becomes obvious that even in differential systems like Ground Based Augmentation Systems (GBAS) which employ BeiDou significant differential user range biases can be observed if different receiver settings are being used at the reference and user receiver This is critical, especially for safety-critical applications if not considered correctly in the integrity budget.

Using DLR's precisely calibrated measurement facility, we will also present an analysis of the transmitted satellite signal power. These kinds of analysis provide furthermore the opportunity to assess a part of the satellite's antenna pattern. Considering the measured power in relation to the boresight angle of the satellite one can measure a cut through the antenna pattern of the satellite and one can assess the antenna symmetry properties. Examples for different satellites will be presented.

\section{MEASUREMENT FACILITY}

For high-quality signal quality analysis, DLR uses a 30m high-gain antenna at ground station Weilheim, Germany (see Figure 3), to capture spectral and I/Q data of GNSS signals. The antenna provides approximately $50 \mathrm{~dB}$ gain in the L-band and guide the signal to a combined L/X-band feed. The feed offers dedicated couplers for various types of polarization and has been configured for right-hand circular polarization (RHCP) signals in the present application. A brief schematic of the setup is presented in Figure 4. 


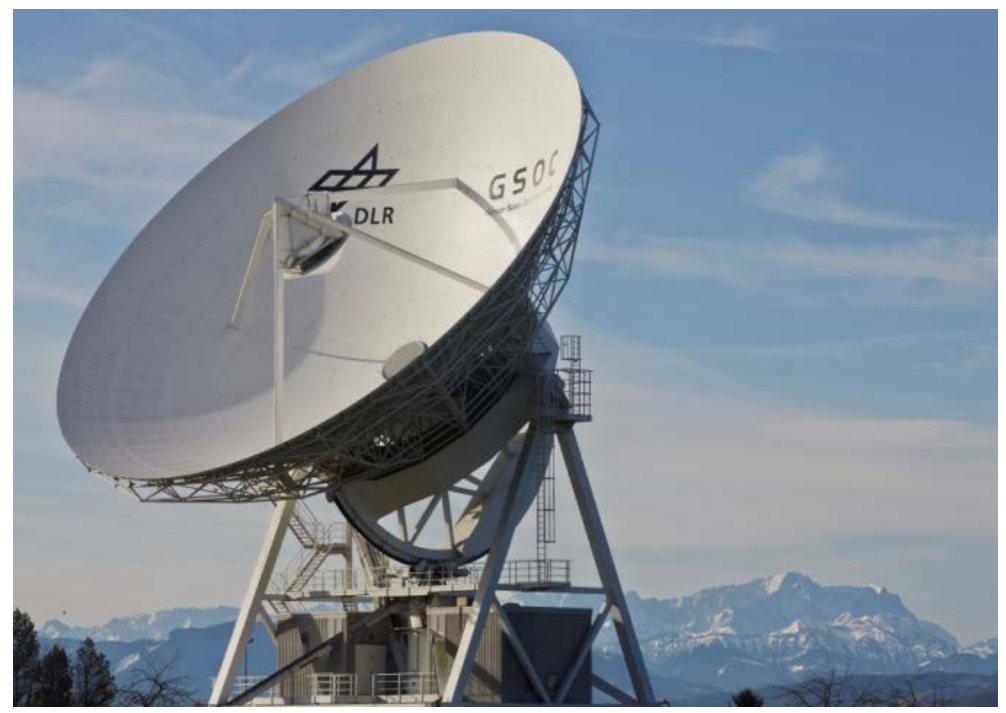

Figure 3 High gain antenna of DLR at Weilheim ground station, Germany operated by the German Space Operation Center (GSOC).

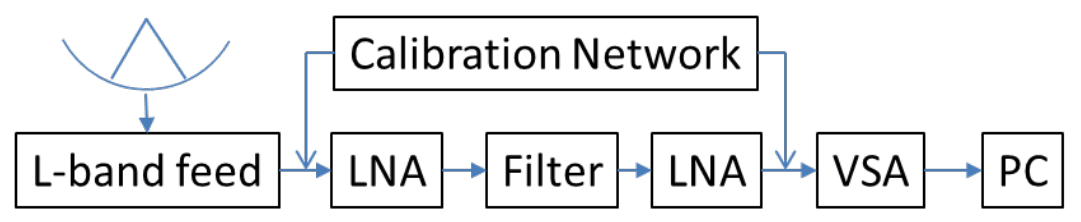

Figure 4 L-band measurement system setup installed in the $30 \mathrm{~m}$ dish at Weilheim, Germany

Behind the feed the signal passes a coupler, low noise amplifier (LNA), filter, and a further LNA. The two LNAs together provide a total amplification of about 70dB, while the filter has a pass-through between $1.1-1.65 \mathrm{GHz}$. The feed in general provides a bit more wideband measurement capability in L-band including the lower frequency range in S-band. After the second LNA another coupler is installed and finally the signal is input to a vector signal analyzer (VSA) for down-conversion and signal recording. With the VSA it is possible to conduct spectral measurements as well as in-phase and quadrature signal acquisitions with a maximum bandwidth of $120 \mathrm{MHz}$, a sampling rate up to 326.4 MHz and a recording length of several hundreds of milliseconds depending on the chosen bandwidth and sampling rate. Note that nowadays GNSS signal transmissions are exclusively present with right hand circular polarization (RHCP). The left hand circular polarization (LHCP) capability of the measurement system is only used for validation of the usually very low cross talk characteristic of the satellite antenna.

The above mentioned two couplers within the signal chain (before and after the LNAs) offer the possibility to inject calibration signals produced by a signal generator, to provide control on the measurement system behavior. This kind of calibration capability covers the signal chain after the feed. The calibration of the $30 \mathrm{~m}$ antenna including the feed is performed using empirical reference data of astronomical sources like Cygnus (Baars et.al. 1966, ITU 2000) and is conducted as described in (Kuz'min 1966, ITU 2000). Detailed information on the overall setup and calibration strategy are provided in Thoelert et.al. (2009, 2013).

The following analysis will benefit from that kind of highly directive antenna by lifting the signal far above the noise floor and avoid incoming environmental distortions from interferences as well as multipath. 


\section{SIGNALS AND SPECTRA}

First, we have a look at the transmitted signal spectra of BeiDou 2 as well as BeiDou 3. Based on I/Q data recording of the VSA over a representative period of one to a few milliseconds, the received signal spectrum is obtained with a selectable resolution. This spectrum has been converted to absolute power spectral density using the total gain determined as part of the overall system calibration. Figure 5 shows as an example the spectrum of the B1 signal transmission of different generations of BeiDou satellites. A comparison between the BDS-2 M6 space craft and two BDS-3 space crafts M2-S and M2 is shown.

Obviously the BDS-2 M6 transmits the former phase-2 signals at $1561.098 \mathrm{MHz}$ center frequency, which contain an open service signal as well as an authorized service component with BPSK(2) modulation each. Both other satellites transmit obviously different signals which are centered at $1575.42 \mathrm{MHz}$.

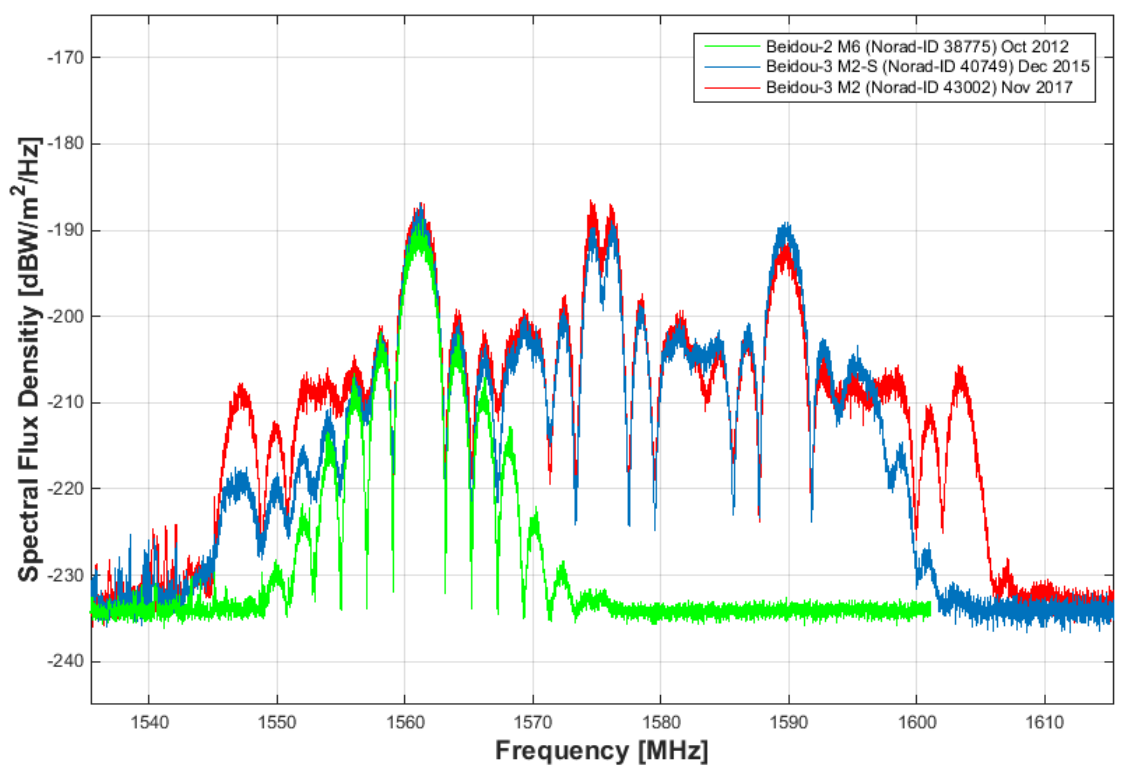

Figure 5 Signal spectra of BeiDou satellites in the B1 band

At this stage it seems necessary to have a more detailed view on the new satellite signal transmissions. For that purpose we consider the B1 signal of BDS-3 M2 separately. Analyzing the spectral shape of the B1 signal one can see main lobes at around $+/-1 \mathrm{MHz}$ and $+/-14 \mathrm{MHz}$ around the center frequency of $1575.42 \mathrm{MHz}$. These lobe can be linked to BOC $(1,1)$ and $\operatorname{BOC}(14,2)$ modulated signal components. Furthermore a small lobe at around $+/-6 \mathrm{MHz}$ can be observed which is the result of a BOC $(6,1)$ modulated signal. Another aspect is the obviously asymmetrically spectral shape regarding the lobes at $+/-14 \mathrm{MHz}$. The reasons can be the frequency response characteristic of the satellite payload or an overlay of an additional signal component located at $-14.322 \mathrm{MHz}$ from the band center frequency. Figure 6 illustrates a composite signal based on the above mentioned signals retrieved from spectral analysis. 


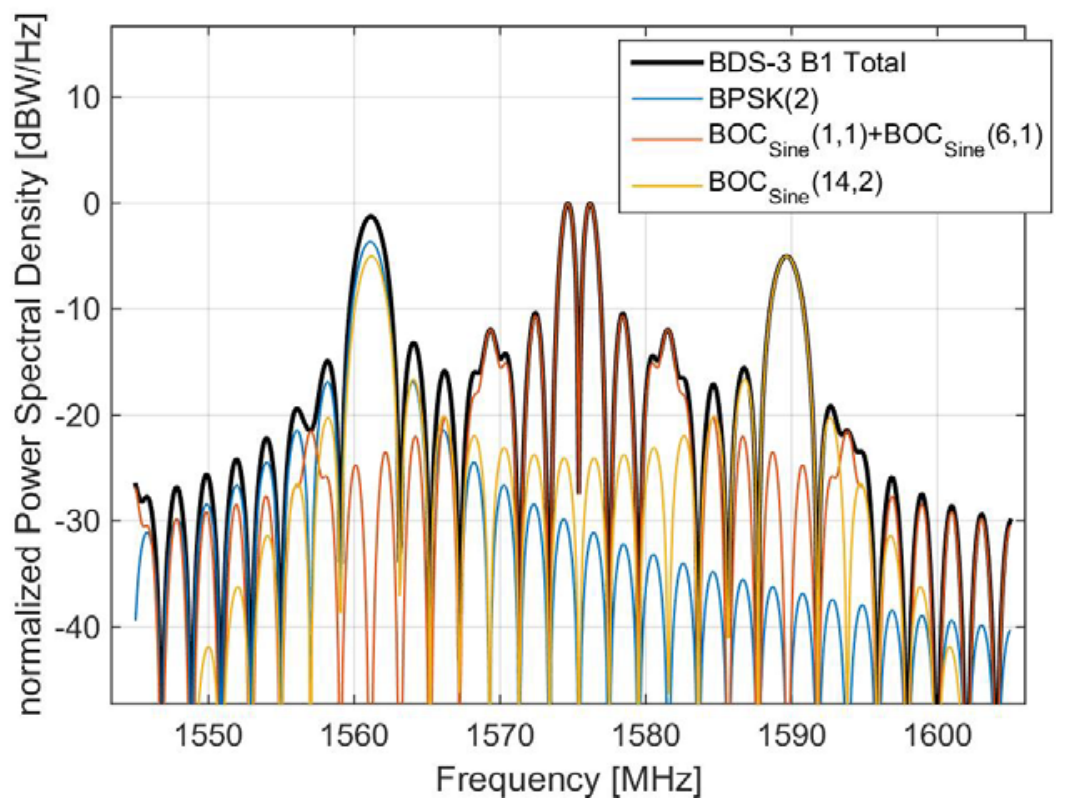

Figure 6: Theoretical spectra of the transmitted signals of a BDS-3 satellite in B1 frequency band.

The analysis of spectral data is limited, since it is only a representation of the absolute magnitude of the signal power over frequency and contains no information about the phase. Based on the captured in-phase and quadrature data further analysis on the transmitted signal components have been performed separately for I and Q channel. Table 1 summarizes the findings. Most of the detected signal components are in line with the literature [Betz 2015, Teunissen and Montenbruck 2017] except the modulation of the B1C open service signal. At the BDS-3 M2 satellite we've found instead of a TMBOC $(6,1,4 / 33)$ a QMBOC $(6,1,4 / 33)$ with $\mathrm{B} 1 \mathrm{C}(1,1)_{\text {pilot }}$ in one channel and $\mathrm{B} 1 \mathrm{C}(1,1)_{\text {data }}$ plus the $\operatorname{BOC}(6,1)_{\text {pilot }}$ in the orthogonal channel of the quadrature modulation.

Table 1: Transmitted signal components in B1-band of BDS-3 M2 satellite

\begin{tabular}{|l|l|l|l|}
\hline Signal Modulation & Service & Service Type & Center Frequency \\
\hline BOC $_{\text {Sine }}(1,1)$ & B1C Data & OS & $1575.42 \mathrm{MHz}$ \\
\hline $\begin{array}{l}\text { BOC }_{\text {Sine }}(1,1)+\text { BOC }_{\text {Sine }}(6,1) \text { as } \\
\text { QMBOC }\end{array}$ & B1C Pilot & OS & $1575.42 \mathrm{MHz}$ \\
\hline BPSK(2) & B1I & OS & $1561.098 \mathrm{MHz}$ \\
\hline BOC $_{\text {Sine }}(14,2)$ & B1A Data & AS & $1575.42 \mathrm{MHz}$ \\
\hline BOC $_{\text {Sine }}(14,2)$ & B1A Pilot & AS & $1575.42 \mathrm{MHz}$ \\
\hline
\end{tabular}

Based on the presented signals in Figure 5 one can derive further information about the evolution of the BeiDou system. The measurements depict also a significant difference in the signal bandwidth between the BeiDou 2 (BDS-2 M6), BeiDou 3 test satellite (BDS-3 M2-S), and the fully operational one (BDS-3 M2). The bandwidth increases from $25 \mathrm{MHz}$, over $50 \mathrm{MHz}$ to $60 \mathrm{MHz}$ over the satellite generations.

Another aspect one can retrieve from the measurement is that a different power sharing between the BOC $(1,1)$ and the BOC $(14,2)$ signals is observed for the M2-S and M2 satellite. Note that at least the open service B1I component of the BeiDou 2 signal is still present in all BeiDou 3 transmissions, both in the test satellites and the fully operational ones. The B1I overlapping with the lower main lobe of the $\operatorname{BOC}(14,2)$ is the reason for the visible spectral asymmetry according to the flux density around the B1 carrier (1575.42 MHz) - $14 \mathrm{MHz}$. 
Not only within the B1 band, but also within the B3 frequency band one can observe some testing experiences of the Chinese. Figure 7 illustrates the measured power spectral density of the received signal on ground of the satellite BDS I2-S (Norad-ID 40938). In the beginning of the satellite's life it transmits a kind of QPSK(10) + BOC $_{\text {Sine }}(15,2.5)$ signal. However, a couple of weeks later the $\operatorname{BOC}(15,2.5)$ components were switched off and still being kept off until today. This is probably part of the testing period and program the S-type satellites are foreseen for. Since the spectra presented in Figure 7 are captured at equivalent satellite elevation it can be seen that after switching off the BOC $(15,2.5)$ components more power is used for the QPSK-like signal.

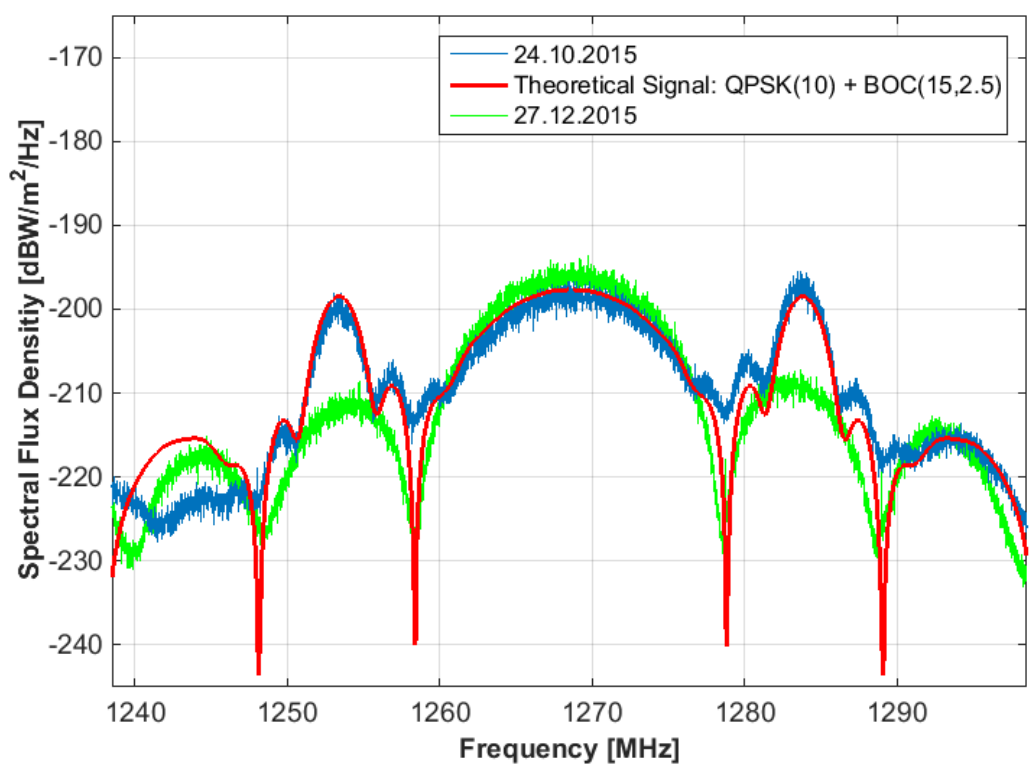

Figure 7 Signal spectra of BeiDou B3 signal

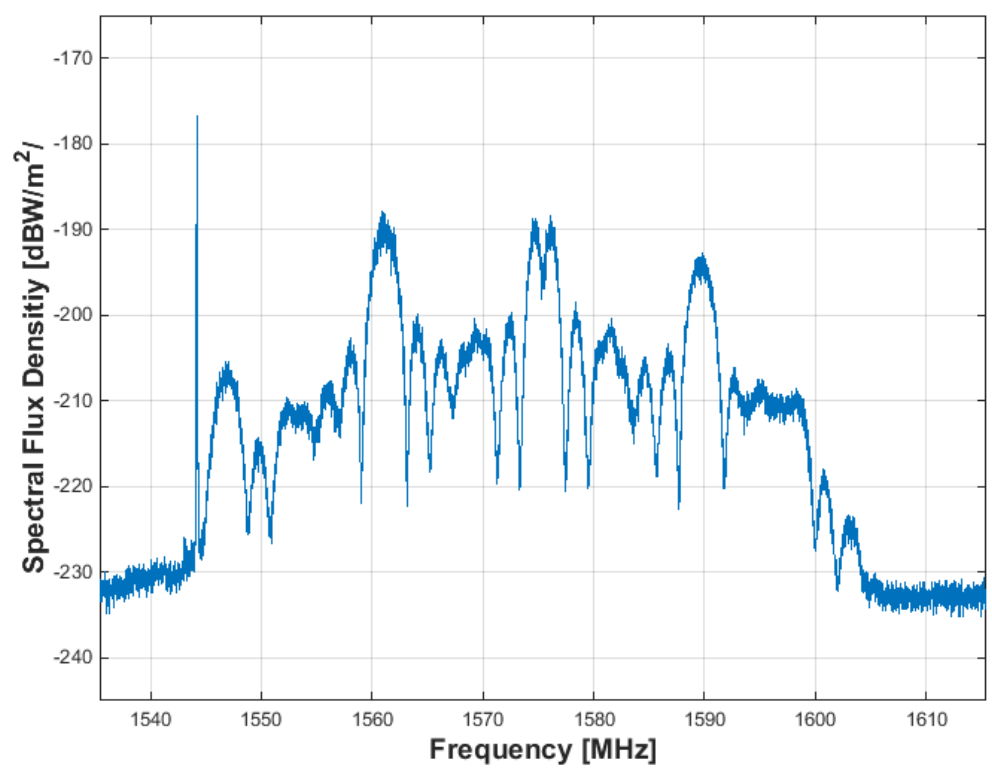

Figure 8 BDS-3 M-13 B1 spectra measured on November 8, 2018

Since the last year the BeiDou 3 M-13 satellite shows a further novelty of the BDS capability. In Figure 8 on the left side of the B1 band a narrow peak can be seen around 1544.2 MHz. This represents a transmission of the search and 
rescue (SAR) payload, which does not belong to the main navigation payload and uses also a different antenna. Also, the satellite BeiDou 3 M-14 carries that kind of SAR payload.

In comparison of the spectra of the BDS-3 M13 B1 signal (Figure 8) with the one from BDS-3 M2 (Figure 5) a different spectral behavior can be seen in the frequency range from $1600 \mathrm{MHz}$ to $1605 \mathrm{MHz}$. The moderate lobe which can be seen around $1603 \mathrm{MHz}$ within the M2 spectra is suppressed in the M13 transmission. Reasons for this adjustment of the signal filtering can be to reduce signal transmission to lower the interference impact on Glonass L1 band and the radio astronomical band at 1610-1614 MHz. However, this is another hint about the flexibility of the BeiDou payload.

\section{ANTENNA PATTERN AND EIRP}

Using DLR's precisely calibrated measurement facility, described in a previous paragraph, analysis of the transmitted satellite signal power has been performed. Based on calibrated spectra and their integration over the according signal bandwidth the total received power can be obtained for each band and converted to the so called equivalent isotropic radiated power (EIRP) using geometrical information about the distance between ground station and satellite. Regarding the later presented results, no atmospheric power losses have been taken into account.

To obtain the EIRP over a wider range of boresight angles with adequate resolution and to be able to provide comparison for minimum received power with SIS-ICD, measurements are typically taken over the full visibility period (rise to set) of a GNSS satellite at representative intervals of about 5 min. Figure 9 presents the estimated EIRP of different BeiDou 3 satellites based on the integration of the measured spectra over the complete transmit bandwidth (50-60 MHz depending on the satellite type). The data recording was performed over the complete overflight for each satellite and two paths are illustrated in Figure 9 (for ascending and descending paths of each satellite).

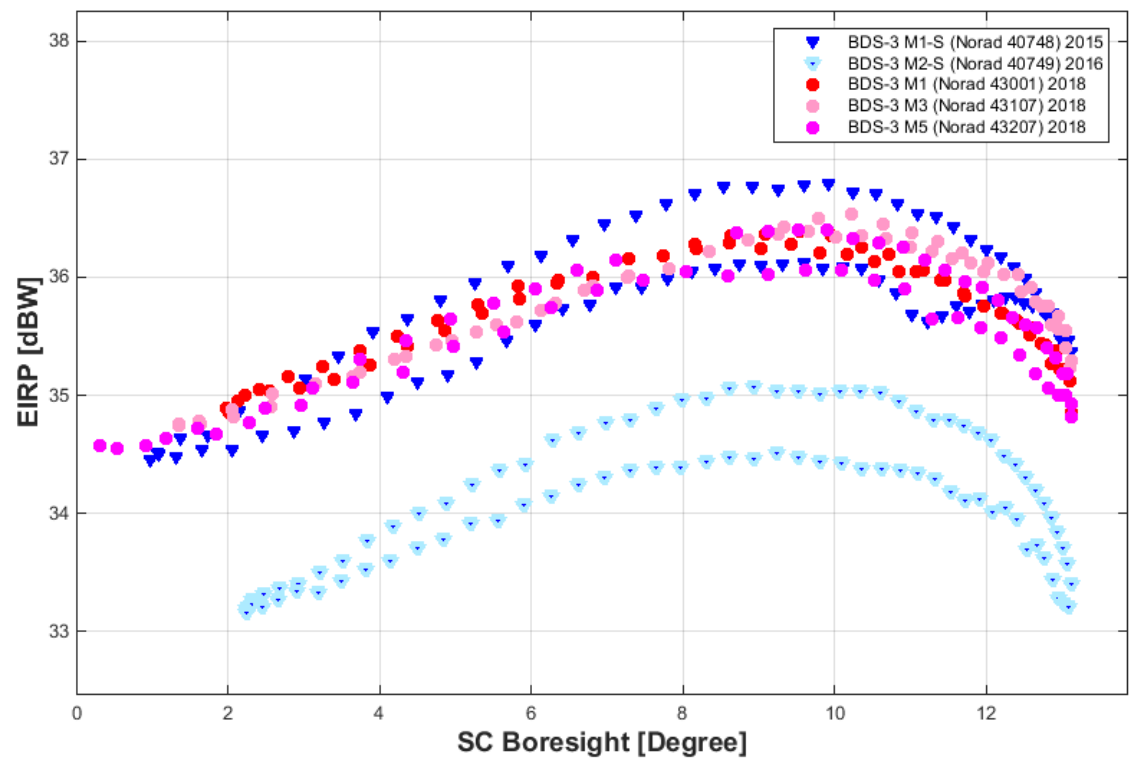

Figure 9 EIRP measurements of BeiDou 3 satellites in the B1-band

For the two measured BeiDou 3 test satellites (M1-S and M2-S) a significant difference in transmit power as well as variation within the ascending and descending paths can be observed. This observed asymmetric patterns result in an unwanted received power variation for users and might lead to performance degradation in some circumstances. This unwanted behavior seems to be corrected for the final BeiDou 3 satellites. The pattern of the M1, M3 and M5 
satellites with the final design seems to be quite symmetric. Further analyses are necessary to confirm that conclusion.

\section{MODULATION}

The recording of in-phase and quadrature samples allows analysis of the constellation diagram and consequently the modulation quality. At a first glance one can see that the constellation diagrams presented in Figure 10 and Figure 11 are different for the S-type satellite from the ones based on the final design. Reasons for the difference can be various, e.g. weaknesses within the synchronization between individual components, different signal interplexing or others. Furthermore the analysis of the I/Q data has shown a change of the implementation of the B1C pilot signal with its components $\mathrm{BOC}(1,1)$ and $\mathrm{BOC}(6,1)$. Within the transmissions of the S-type BDS-3 satellites a TMBOC could be confirmed [Bravaro 2015]. In contrast the authors can verify via a correlation test using ideal BOC $(6,1)$ chips the presence of a QMBOC modulation regarding the B1C pilot component in the BDS-3 M2 B1 satellite transmission. This finding is also in line with the published B1C ICD [ICD B1C 2017]. The change of the B1C modulation scheme also yields in a change of the constellation diagram. However, this all shows again that the Chinese updated their satellite platform design from the BDS-3 test phase up to the final stage.

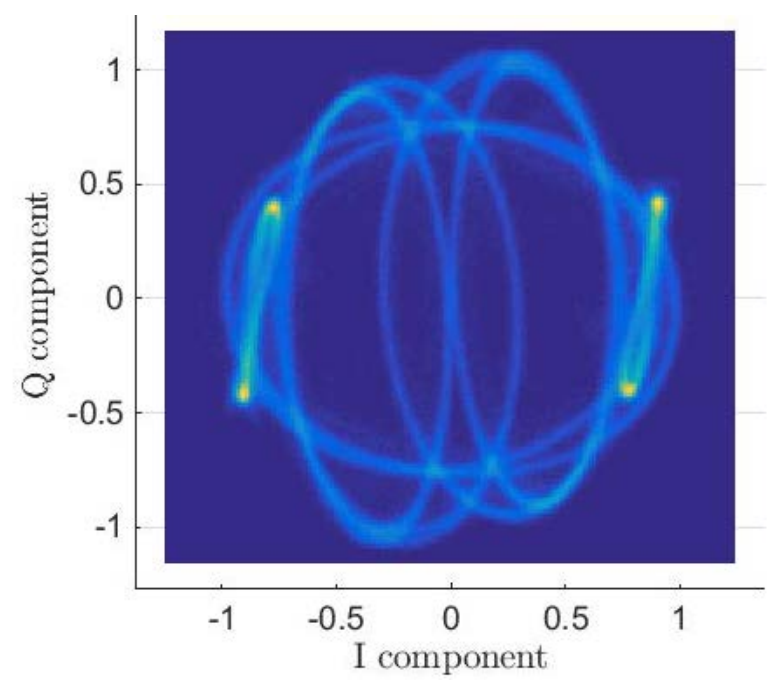

Figure 10 BeiDou 3 I2S (Norad-ID: 40938) B1 constellation diagram

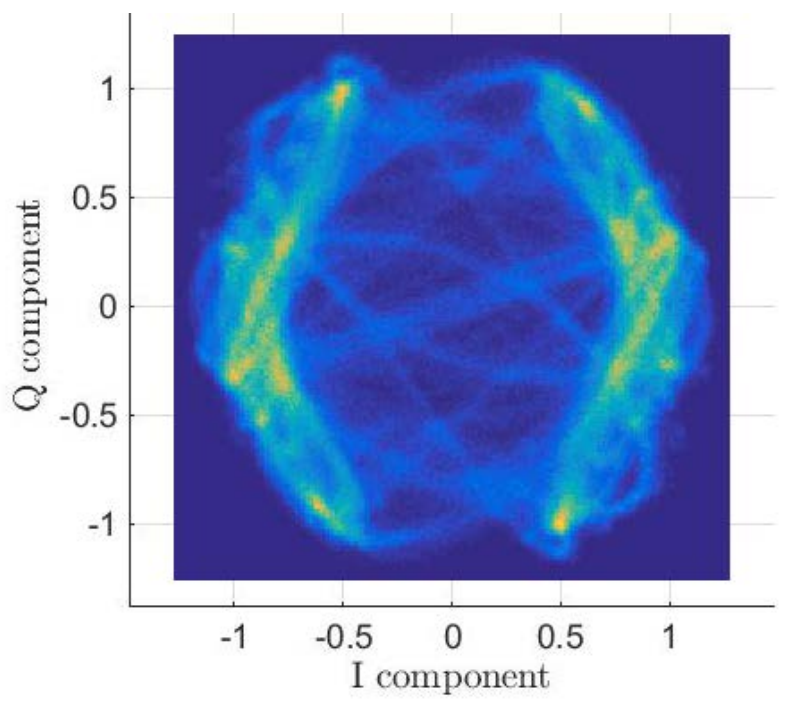

Figure 11 BeiDou 3 M3 (Norad-ID: 43002) B1 constellation diagram 


\section{SIGNAL DISTORTIONS}

In this section we want to quantify the impact of the payload distortions on the navigation performance obtained by an ideal GNSS receiver. The S-curve bias information [Van Dierendonck 1992, Soellner 2008, Pagot 2015] presented in the following is useful for every kind of differential GNSS application, e.g., augmented applications like GBAS. Usually in that kind of application more than one receiver is used with a high possibility of using different settings for the receivers. In many applications not only the settings are different but also the complete equipment (receiver, front-end, antenna and corresponding filter masks...). For all these applications the bias based on different settings caused by the signal deformations are relevant.

The analysis is based on the captured I/Q samples. In order to provide results which are only based on the satellite payload characteristic itself, it is important not only to compensate the receive measurement system behavior, but also the influence of the propagation path, i.e.in particular the ionosphere. The correction of the ionosphere is performed using high accurate total electron content (TEC) maps provided by DLR, Neustrelitz, Germany [IMPC DLR].

In general the corresponding acquisition and tracking performance of a navigation receiver is based on the cross correlation function (CCF). The correlation between the received signal and an ideal signal replica can be expressed as:

$\operatorname{CCF}(\varepsilon)=\int_{0}^{T p} s_{\text {meas }}(t) \cdot s_{\text {Replica }}^{*}(t-\varepsilon) d t$,

with $T_{p}$ being the coherent integration time (in case of B1C signal $10 \mathrm{~ms}$ ), $\mathrm{s}_{\text {meas }}(\mathrm{t})$ denotes the measured signal, $\mathrm{S}_{\text {Replica }}(\mathrm{t})$ the replica signal, and $\varepsilon$ the delay between the signals.

This definition provides the benefit to separate the satellite transmit distortions of interest from receiver distortions, e.g. caused by the receiver frontend. Furthermore the correlation provides the feature of a matched filter, which results in a separation with respect to the other transmitted signal components. Figure 12 shows the cross-correlation function of the receive signal and an ideal B1C pilot replica. Note that in the following analysis for the B1C pilot component only the $\mathrm{BOC}(1,1)$ signal part is considered.

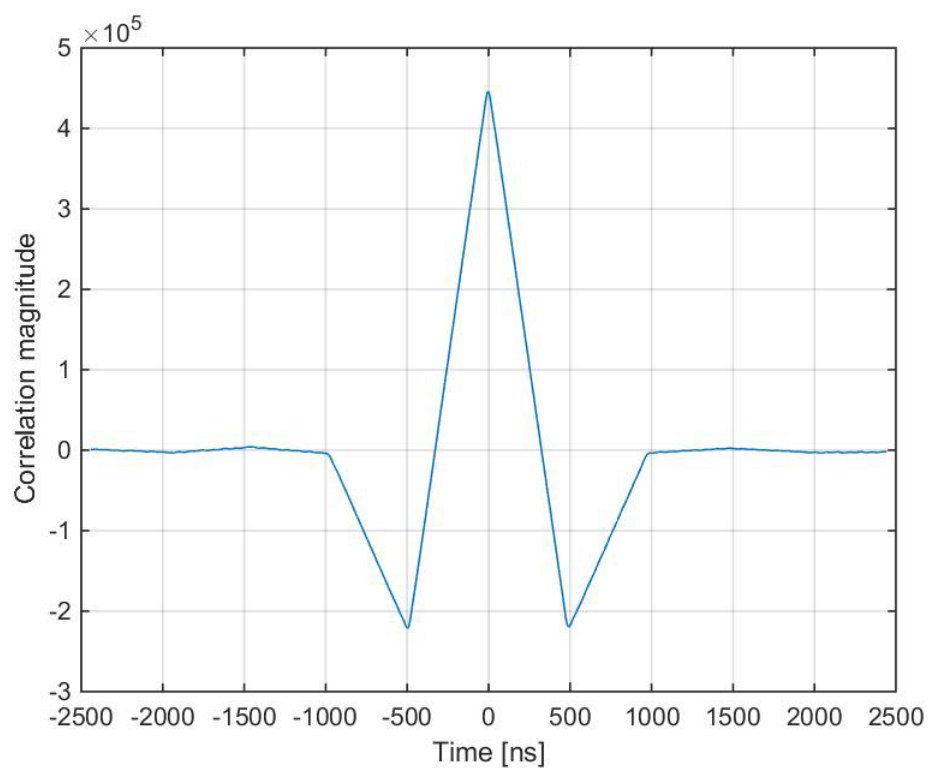

Figure 12: Cross correlation function of received signal (60 MHz filtered) and ideal B1C pilot replica. 
Considering an "early minus late" non-coherent discriminator and sliding it over the correlation function one gets the so called S-curve

$\operatorname{Scurve}(\varepsilon, \delta)=\left|\operatorname{CCF}\left(\varepsilon+\frac{\delta}{2}\right)\right|^{2}-\left|\operatorname{CCF}\left(\varepsilon-\frac{\delta}{2}\right)\right|^{2}$,

with $\delta$ being the early - late spacing in chip duration $\mathrm{T}_{\mathrm{c}}$, usually chosen for a $\mathrm{BOC}(1,1)$ signal between 0 and $\mathrm{T}_{\mathrm{c}} / 2$ [Betz 2015].

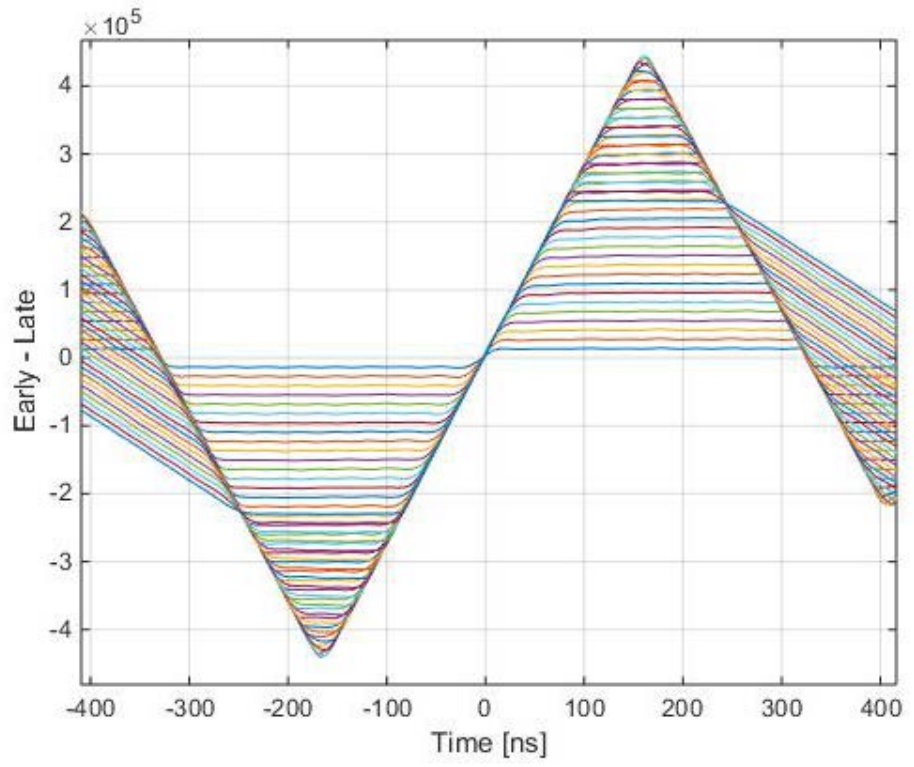

Figure 13: S-curve for various early-late correlator spacings based on CCF for B1C pilot.

The navigation receiver obtains the lock-point $\varepsilon_{\text {lock }}$ by the zero-crossing of the code discriminator function $S(\varepsilon, \delta)$ (i.e., the S-curve) which consequently can be defined as

$S_{\text {lock }}\left(\varepsilon_{\text {lock }}, \delta\right)=0$.

For a non-distorted signal and consequently for an ideal CCF the zero crossing for different early-late spacings would be zero. However, in reality, due to signal distortions, the lock points for different spacing are different as can be seen in Figure 13. 


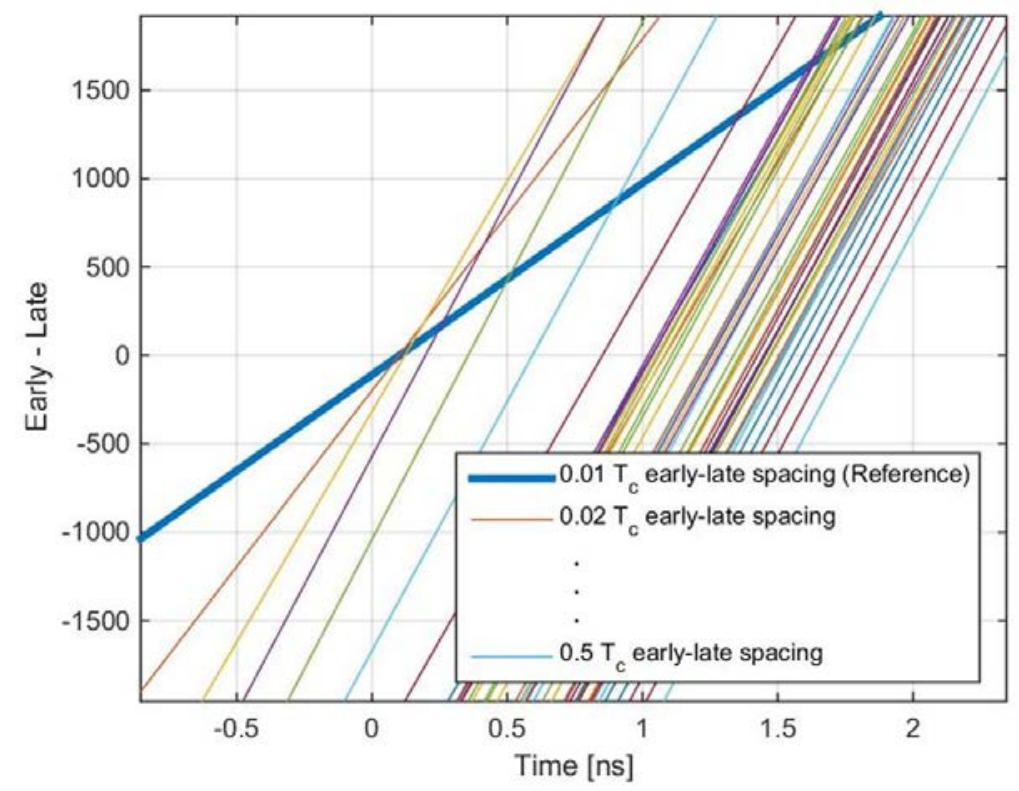

Figure 14: Extract of the S-curve for various early-late correlator spacings based on CCF around the zero crossing.

The resulting S-curve bias can now be determined by calculating the difference of each lock-point with respect to a defined reference lock-point. For the presented example the reference lock-point is chosen for an early-late spacing of $0.01 \mathrm{~T}_{\mathrm{c}}$, which is also highlighted within Figure 14.

Figure 15 shows the resulting S-curve bias for a $60 \mathrm{MHz}$ filtered received signal using an ultra-narrow correlator spacing of $0.01 \mathrm{~T}_{\mathrm{c}}$ as a reference.

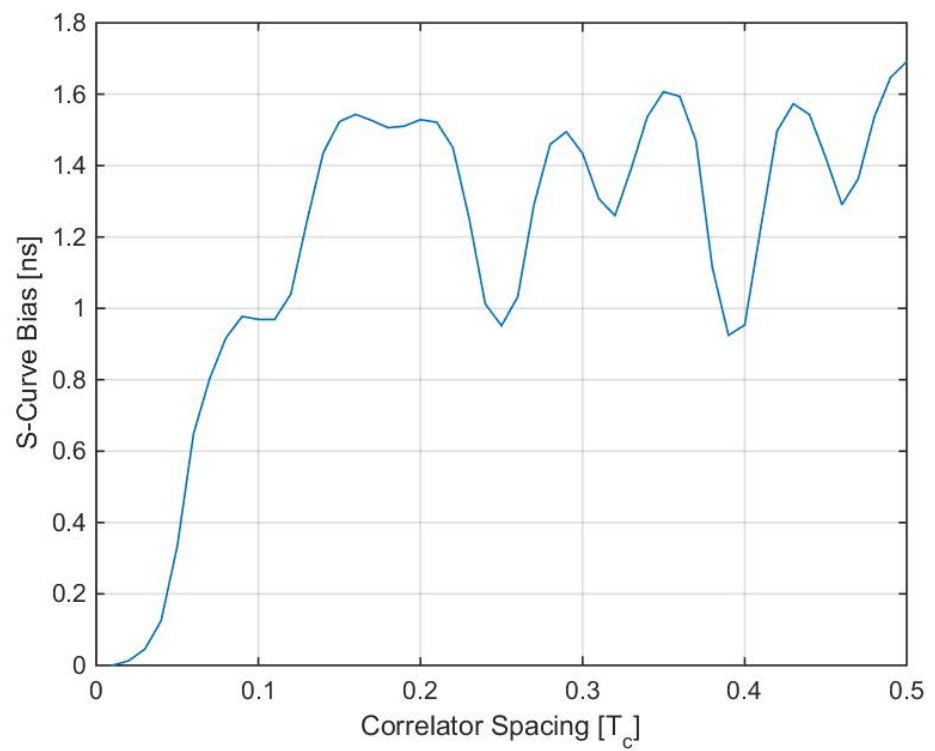

Figure 15: S-curve bias based on $0.01 \mathrm{~T}_{\mathrm{c}}$ reference S-curve zero crossing and $60 \mathrm{MHz}$ filtered receive signal.

The variation based on the signal deformation is clearly visible. Now, one can extend this analysis varying the receive signal bandwidth, since often different receivers are using different analog and digital frontend filter settings resulting in various bandwidths. The reference receiver setting is assumed to be the already mentioned ultra-narrow correlator spacing of $0.01 \mathrm{~T}_{\mathrm{c}}$ and an input bandwidth of $20 \mathrm{MHz}$ (two-sided). Figure 16 and Figure 17 show the 
resulting S-curve bias for varying the correlator spacing from 0.01 to 0.5 and the input bandwidth from $4 \mathrm{MHz}$ to 60 $\mathrm{MHz}$ for both the B1C pilot and data signal component, respectively.

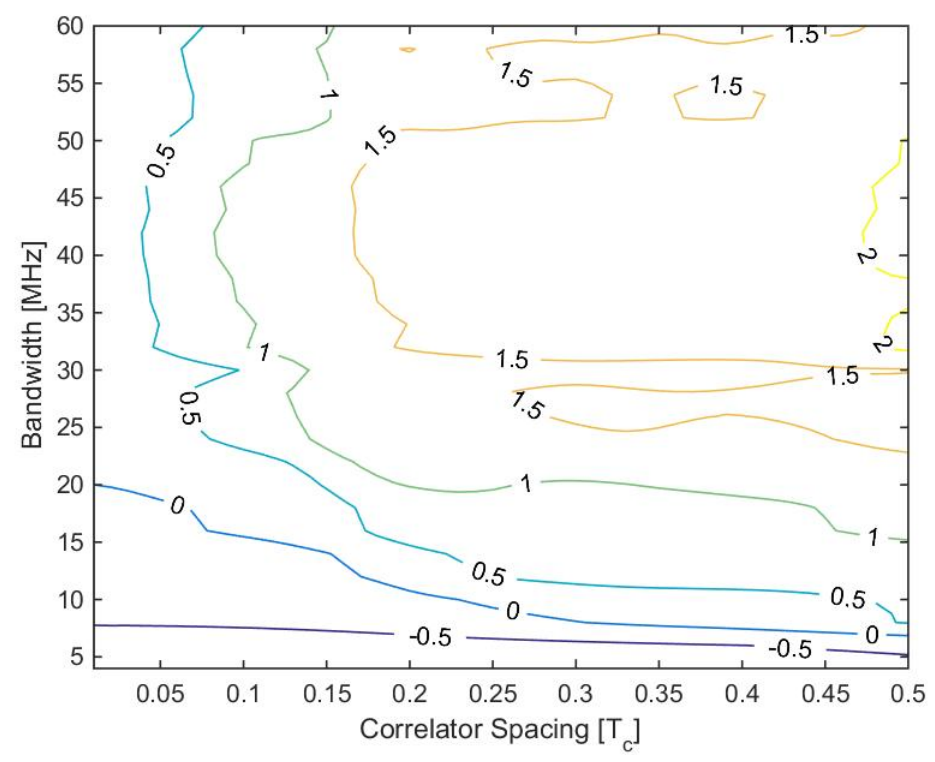

Figure 16: S-curve bias in [ns] for the B1C pilot component versus bandwidth and correlator spacing.

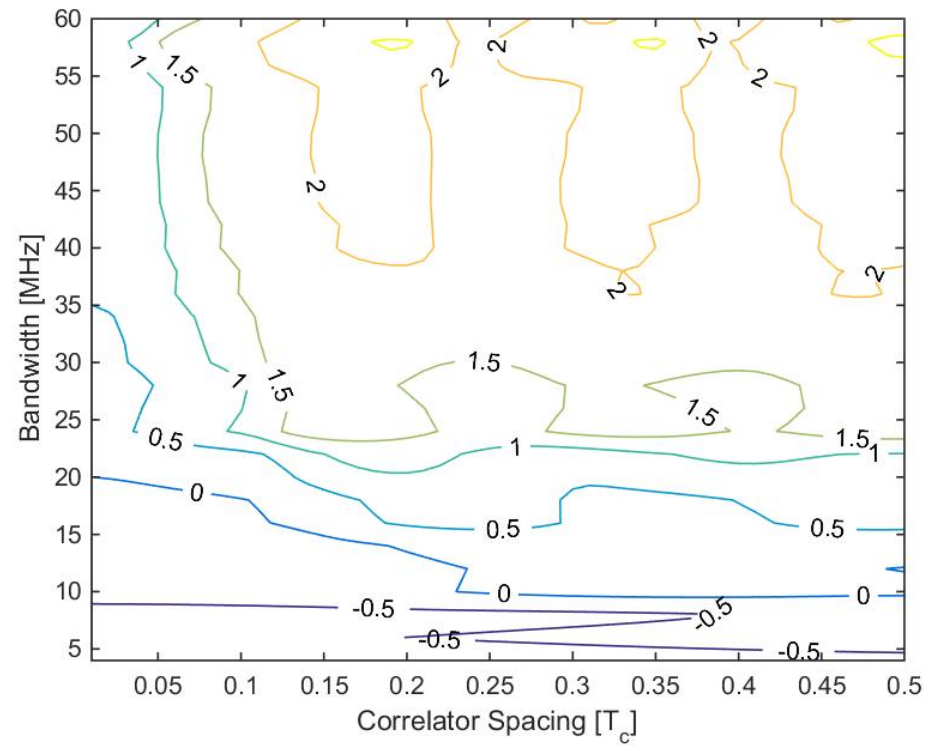

Figure 17: S-curve bias in [ns] for the B1C data component versus bandwidth and correlator spacing.

The resulting bias for the applied reference setting is in the range from $-0.5 \mathrm{~ns}$ up to $2 \mathrm{~ns}$. Translated to resulting pseudo-range measurement errors the variations are in the range from $-15 \mathrm{~cm}$ to $60 \mathrm{~cm}$. This means in a differential GNSS application using two receivers with different configurations for correlator spacing and input bandwidth a bias of up to $75 \mathrm{~cm}$ can occur for the range measurement for the same satellite. The results indicate the need to carefully choose the receiver settings in a differential GNSS application. 


\section{CONCLUSION}

This paper shows a significant modification from the Chinese regional BeiDou 2 navigation system into the global BeiDou 3 system. The amount of provided services, the signal frequencies, bandwidth and signal power of BeiDou 3 satellites have been improved significantly comparing to the old system. This provides a higher compatibility and competitiveness regarding other existing GNSS like GPS or Galileo. The observed filter bandwidth of approximately $60 \mathrm{MHz}$ for the B1 frequency band of the BeiDou3 system in combination with a fully digital and programmable signal generation unit also provides the possibility of later changes of the provided services (signal flexibility). The use of this flexibility has been already demonstrated, e.g. with the modification of the filtering from BDS-3 M2 to BDS-3 M13.

For the transition from BeiDou 2 into BeiDou 3 five test satellites (indicated by an "S" in their names) have been implemented in space, which have in general, full operational capability, but the signal transmission and payload characteristic is slightly different from the later final satellites. Based on the measured higher bandwidth and signal power as well as a more symmetric antenna pattern within the B1 frequency band, we can confirm that a design change of the satellite platform has been performed to improve the payload performance.

The analysis of the signal deformations of the open service components BOC(1,1) data and pilot show variations of the S-curve bias of up to $75 \mathrm{~cm}$ which is comparable to other GNSS systems [Thoelert 2014]. Further analysis is necessary to confirm the amount of signal deformations also for other satellites of the BeiDou 3 system.

\section{ACKNOWLEDGEMENT}

The authors want to thank their colleagues from the German Space Operations Center (GSOC) for supporting and operating the high gain antenna at DLR's ground station Weilheim, Germany. Furthermore, the authors thank their colleagues from DLR Neustrelitz, for the provision of high-resolution TEC maps.

\section{REFERENCES}

Baars J, Genzel R, Pauliny-Thoth I, Witzel W (1977) The absolute spectrum of Cas A: an accurate flux density scale and a set of second calibrators. Astron Astrophys 61(1):99-106

Betz JW (2015) Engineering Satellite-Based Navigation and Timing. Wiley

Bravaro M, Curran J, Fortuny J (2015) First Signals of BeiDou Phase 3 Acquired at Ispra, Italy. GPS World, Vol. 26 (9)

Cameron A (2015) New BeiDou TMBOC Signal Tracked; Similar to Future GPS L1C Structure. GPS World, Vol. $26(10)$

Interface Control Document (ICD) - Open Service Signal B1C (Version 1.0) by China Satellite Navigation Office, 2017

Ionosphere Monitoring and Prediction Center, German Aerospace Center - DLR, Neustrelitz, Germany, https: \limpc.dlr.de

Kuzmin AD, Salomonovich AE (1966) Radioastronomical methods of antenna measurements. Academic Press

Pagot JB, Thevenon P, Julien O, Gregoire Y, Amarillo-Fernandez F, et al.. (2015) Estimation of GNSS Signals' Nominal Distortions from Correlation and Chip Domain. ION ITM 2015, Jan 2015, Dana Point, California, USA.

Partridge MD and Dafesh PA (2001), Code Power Measurement Methodology for GPS Block IIR-M and IIF Onorbit Test Procedures., Proc. ION GPS, pp. 2764-2772, Salt Lake City, UT, USA. 
RECOMMENDATION ITU-R S.733-2 (2000) Determination of the g/t ratio for earth stations operating in the fixedsatellite service. 2000

Soellner M, Kurzhals C, Hechenblaikner G, Rapisarda M, Burger T, Erker S, Furthner J, Grunert U, Meurer M, Thölert S (2008) GNSS Offline Signal Quality Assessment. In: Proc. ION GNSS 2008, Page 909-920. 21. International Technical Meeting of ION GNSS 2008, 16-19 September, Savannah (USA).

Thoelert S, Erker S, Meurer M (2009) GNSS signal verification with a high gain antenna—calibration strategies and high quality signal assessment. In: ION ITM 2009. Portland, pp 2896-2909

Thoelert S, Furthner J, Meurer M (2013) GNSS survey - Signal Quality Assessment of the latest GNSS satellites. In: ION ITM 2013. San Diego, pp 608-615

Thoelert S, Vergara M, Enneking C, Antreich F, Meurer M, Brocard D, Rodriguez CS (2014), Characterization of Nominal Signal Distortions and Impact on Receiver Performance for GPS (IIF) L5 and Galileo (IOV) E1 /E5a Signals, ION GNSS, Tampa, FL, USA

Thoelert S, Hauschild A, Steigenberger P, Langley R (2017) GPS IIR-M L1 Transmit Power Redistribution: Analysis of GNSS Receiver and High-Gain Antenna Data. In: ION GNSS 2017. ION GNSS, 25.-29. Sep. 2017, Portland, USA

Teunissen PJG and Montenbruck O (Eds.) (2017) Springer Handbook of Global Navigation Satellite Systems, Springer International Publishing, ISBN 978-3-319-42926-7

Van Dierendonck AJ, Fenton P, Ford T (1992) Theory and Performance of Narrow Correlator Spacing in a GPS Receiver. NAVIGATION, Journal of the Institute of Navigation, Vol 39, No 3, Fall 1992

Vergara M, Sgammini M, Thoelert S, Enneking C, Zhu Y and Antreich F (2016) Tracking Error Modeling in Presence of Satellite Imperfections. Navigation - Journal of the Institute of Navigation 63(1):3-13, DOI: 10.1002/navi.129

Wu Y, Liu X, Liu W, Ren J, Lou Y, Dai X, Fang X (2017) Long-term behavior and statistical characterization of BeiDou signal-in-space errors. GPS Solutions, Vol. 21(4), pp 1907-1922

Xiao W, Liu W, Sun G (2016) Modernization milestone: BeiDou M2-S initial signal analysis. GPS Solutions, Vol. 20 (1), pp 125-133

Yang Y, Tang J, Montenbruck O (2017) Chinese Navigation Satellite Systems. Springer Handbook of Global Navigation Satellite Systems. Teunissen PJ and Montenbruck O, Eds. Springer International Publishing, p. 280

Zhou R, Hu Z, Zhao Q, Li P, Wang W, He C, Cai C, Pan Z (2018) Elevation-dependent pseudorange variation characteristics analysis for the new-generation BeiDou satellite navigation system. GPS Solutions, Vol 22(:60) 